\title{
Fast Localization of Impact Damage on Woven CFRP Based on Sparse Microwave Imaging
}

\author{
Ruslee Sutthaweekul ${ }^{\mathrm{a}, 1}$ and Gui Yun Tian ${ }^{\mathrm{b}}$ \\ ${ }^{a}$ Department of Electrical and Computer Engineering, Faculty of Engineering, King \\ Mongkut's University of Technology North Bangkok, Thailand, 10800 \\ ${ }^{\mathrm{b}}$ School of Engineering, Newcastle University, Newcastle, UK, NE1 7RU
}

\begin{abstract}
Microwave open-ended waveguide scanning has been proved to be a promising NDT technique for imaging of woven carbon fiber reinforced plastic (CFRP) with impact damages. However, it uses a conventional C-scan technique, which is excessively time-consuming and therefore it is not practical for defect detection in a large area. Moreover, without proper feature extraction, the appearance of woven texture within the impact damage area opposes to the localization of the impact. This work proposes a novel framework for fast impact damage localization by means of discrete sparse measurement. Also, the localization accuracy of sparse measurement is improved by mitigating effect of woven texture using principal component analysis (PCA) for feature extraction. The performance of the proposed methods is evaluated by sets of incremental measurements. The performance of the proposed system is and evaluated by localizing the impact damage of 100 non-uniform sparse sampling sequences. Results show that our proposed technique can accurately localize the impact damage with number of samples less than $10 \%$ of full measurements, giving approximately 10 times faster than that of the conventional C-scan.
\end{abstract}

Keywords. open-ended waveguide, CFRP, PCA, sparse measurement, impact damage, localization

\section{Introduction}

Woven carbon fiber reinforced plastic (CFRP) has been developed and widely used in advanced engineering structures such as aerospace, automobile, and sports equipment. It is due to the superiority in stiffness to weight ratio property. Though CFRP is easily damaged by impact and dropped tools even at very low impact energy [1]. Among electromagnetic NDT techniques for composites, microwave open-ended waveguide is one of the promising techniques for inspection of woven CFRP [2,3]. The ability of this technique has been demonstrated by reconstructing high resolution images of texture and impact damage on woven CFRP [4]. However, this technique requires a traditional full area scan (C-scan) in near-field, which is time consuming and not practical for defect detection in a large area (e.g., aircraft fuselage).

To overcome the challenge of time consuming, the technique of compressed sensing (CS) were introduced [5,6]. Works in [5], we applied a CS technique based on discrete

${ }^{1}$ Department of Electrical and Computer Engineering, Faculty of Engineering, King Mongkut's University of Technology North Bangkok, Thailand, 10800; E-mail: ruslee.s@eng.kmutnb.ac.th. 
cosine transform (DCT). Results shows that the quality of the reconstructed images is acceptable with the sparse sampling rate of only less than $20 \%$ (approximately 5 time quicker than the full measurement). Despite that, the CS requires intensive computation power and therefore it may not suitable for real-time process. Yet, without a proper feature extraction technique, the presence of woven texture within the damage area diminishes detection and localization abilities of impact damage. So far, there no comprehensive research that investigated feature extraction from spectral responses of woven CFRP based on a forward model approach. It is due to the complex of woven CFRP, which involves the weaving structure of inhomogeneous materials with different properties. Alternatively, a statistical approach based on machine learning such as principal component analysis (PCA) has demonstrated ability in solving this type of challenges. In the microwave NDT study, an open-ended rectangular waveguide probe has been employed for characterization of steel corrosion in early stages [7]. The PCA was effectively applied for feature extraction of hidden progressive corrosions with the elimination of the coating layer influence.

In this paper, it is the first time that we investigate feature extraction of impact damage on woven CFRP based on PCA of wideband signal responses (18-26.5 GHz). A principal component exhibiting damage feature with least inference of woven texture will be selected for feature extraction of the impact damage. The performance of proposed PC based feature extraction will be demonstrated by sets of sparse measurement. The rest of this paper is organized as follows. Section 2 describes PCA for feature extraction, procedures for impact damage localization, CFRP sample and measurement setup. Section 3 shows and discusses the results of PCA feature extraction and its localization performance. Section 4 concludes the major findings and the potential future works are discussed.

\section{Materials and Methods}

The framework of fast localization of impact damage on woven CFRP based on sparse measurement is shown in Figure 1. Prior to the impact damage localization, an offline PC model has to be obtained through PCA. The detailed descriptions of each process are described in the following sub-sections.

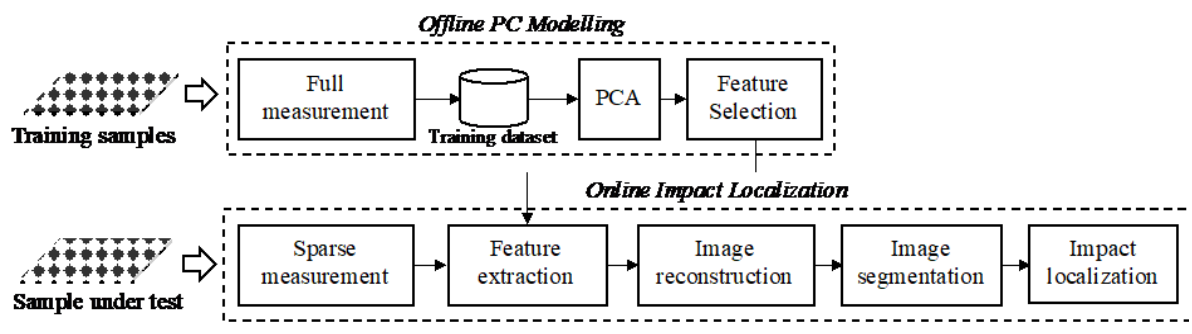

Figure 1. Block diagram for impact damage localization from sparse measurement

\subsection{Obtaining a Principal Component Model for Impact Damage Feature Extraction}

A principal component (PC) model for impact damage extraction has to be obtained using PCA. PCA has been widely used for feature extraction reducing data dimensions 
and preserving prominent features [8,9]. The PCA process for feature extraction consists of two major steps: training and feature selection. In training, covariance matrices of training data sets are formed by subtraction of mean value from each of the data dimensions resulting in a dataset of zero means. The covariance matrix is decomposed to retrieve a matrix of eigenvectors $e_{i}$ and eigenvalues $\lambda_{i}$. The eigenvectors ranked in descending order of coherent eigenvalues are called principal components (PCs). In feature selection, only high-rank PCs are considered for feature extraction. The projection results of each PC will be visually compared and the PC having the results most correlated to the interesting feature will be selected for feature extraction.

\subsection{Fast Localization of Impact Damage}

Sparse sampling data are obtained for estimation of the impact location. The processing for each sampling iteration is explained in a flowchart in Figure 2. Initially, the sampling data of four scanning corners are used to fill up the unknown area and form a feature image through Natural-neighbor interpolation technique [10,11]. After that, K-means clustering, with $K=2$ [12], is used for binary image segmentation and obtains the impact damage area based on the pixel illuminations. Next, the centroid of the impact area is calculated and used to represent the impact center. If the current impact location does not converge to that of the previous iterations and there are more sampling locations, the whole process is repeated with a new sampling data from the next scanning location.

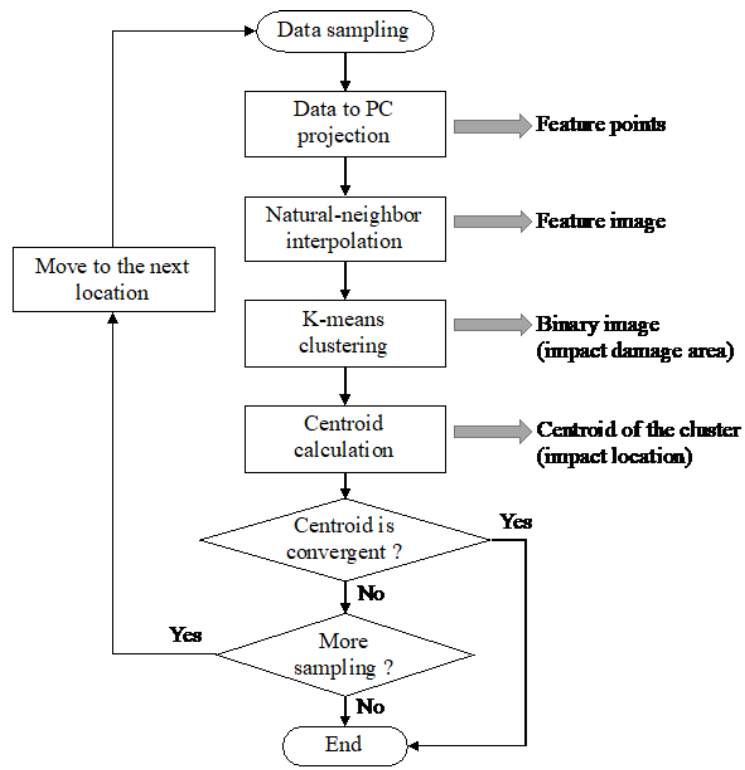

Figure 2. Flowchart of impact localization based on incremental sparse sampling

\subsection{Samples and Measurement Setup}

The impact damage on CFRP sample is produced by a drop test equipment with a hemispherical bumper head of $20 \mathrm{~mm}$ in diameter [4]. It is known that the common defects of low energy impact include surface dent, matrix crack, delamination and fiber 
breakage [13]. We choose the $6 \mathrm{~J}$ impact sample as the training sample because the damage area is large enough for statistical inputs in PC learning process.

The main equipment in the experiment consists of an X-Y scanner (High-Z S-720), a vector network analyzer or VNA (Agilent PNA E8363B) and an open-ended waveguide probe (WR-42). Before the experiment, the tools are calibrated and configured according to the measurement parameters given in Table 1. A computer is used to control the scanning route and store the data for further analysis.

Table 1. Measurement parameters for obtaining training dataset and localization experiments

\begin{tabular}{ll}
\hline \multicolumn{1}{c}{ Measurement Parameter } & \multicolumn{1}{c}{ Value } \\
\hline Operating frequency & $18.0-26.5 \mathrm{GHz}$ \\
Number frequency points & $1601(\Delta \mathrm{f}=3.5 \mathrm{MHz})$ \\
Probe & Open-ended rectangular waveguide probe No. WR-42 \\
Scanning step size $(\triangle \mathrm{x}$ and $\triangle \mathrm{y})$ & $0.5 \mathrm{~mm}$ \\
Probe lift-off & $1 \mathrm{~mm}$ \\
Delay between sample & $1 \mathrm{~second}$ \\
Scan areas & $50 \times 50 \mathrm{~mm}^{2}$ for training dataset and 30x60 $\mathrm{mm}^{2}$ for localization \\
& experiments \\
\hline
\end{tabular}

\section{Results and Discussions}

\subsection{Selection of Principal Component for Impact Damage Feature Extraction}

A set of principal components are calculated as described in Section 2.1. Only the first six principal components (PC1-PC6) are chosen to be analyzed according to the sufficiency of the cumulative percentage of variance described in Table 2.

Table 2. Percentage and Cumulative Percentage of Variances of PC1-PC6

\begin{tabular}{lrrrrrr}
\hline & PC1 & PC2 & PC3 & \multicolumn{1}{c}{ PC4 } & \multicolumn{1}{c}{ PC5 } & PC6 \\
\hline Percentage of variance & 44.66 & 23.03 & 16.46 & 9.59 & 3.14 & 1.67 \\
Cumulative \% variance & 44.66 & 67.69 & 84.15 & 93.74 & 96.88 & 98.55 \\
\hline
\end{tabular}

The reconstructed feature images based on the projection of PC1-PC6 to the training data of full measurement are displayed in Figure 3. Although damages are clearly recognized in both PC1 and PC6, it can be seen that the images from PC1 to PC5 are strongly affected by woven textures, while the PC6 represents an area of impact damage with the least background of woven texture. Hence in this case, only the PC6 is selected as an offline PC model for feature extraction of the impact damage.
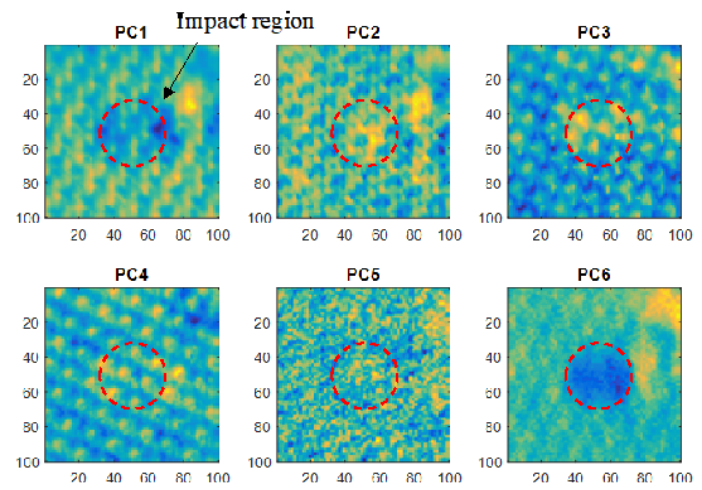

Figure 3. Principal component: PC1-PC6 projected feature images 


\subsection{Performance of Fast Impact Damage Localization}

In each scanning sequence, the data are sampled and processed by the procedures described in Section 2.2. The number of sampling points is in the range from 4 to 720 samples in a sequence. The results of PCA feature is compared with the average magnitude (AM) feature, which is the common feature for microwave and millimeter wave imaging [6]. Figure 4 shows an example of processing results obtained by one scan (out of 100 scans). Only 4 steps $(\mathrm{P}=4, \mathrm{P}=72, \mathrm{P}=288$, and $\mathrm{P}=720$ ), with significant changes in the results, are selected to display. At $\mathrm{P}=4(0.05 \%$ of full measurement $)$ : impact damage does not reveal in both AM and PCA feature images. At $\mathrm{P}=72(0.98 \%$ of full measurement): a small area of impact damage is visible in both AM and PCA feature images. The PCA feature image shows a cleaner impact damage without the edge interference. As result, the calculated centroid of clustered AM is somewhere around the top edge of the sample, whereas the centroid of clustered PCA is confined within the impact area. At $\mathrm{P}=288$ (3.90\% of full measurement): The AM feature image shows fragmented impact area because of the presence of woven texture. The centroid of clustered AM is still distant to the actual impact location. The centroid of clustered PCA moves closer to the actual impact location. At $\mathrm{P}=720$ (9.75\% of full measurement): the centroid of AM feature moves closer to the actual impact location, whereas the centroid of the PCA feature is convergent as it is already at the location of the actual impact.

The performance of PCA and AM feature for impact damage localization is evaluated by a plot of average distance error over 100 sparse sampling sequences shown in Figure 5. It can be seen that PCA gives higher distance error at the beginning. However, the distance error of PCA goes below that of the AM after around 26 locations are sampled. Then, unlike the AM feature, PCA rapidly approaches zero error at around $>720$ samples or about $10 \%$ of the full measurement.

\section{Conclusions}

In this work, a novel technique of feature extraction for fast localization of impact damage on woven CFRP is proposed. Our major findings in this work can be summarized as follows.

- $\quad$ PCA demonstrates an ability to characterize impact damage on of woven CFRP with mitigation of the background woven texture.

- Compared to AM feature, the impact location determined from PCA feature is more stable during the progressive area scan.

- Centroid of segmented damage area gives better localization accuracy than the minimum value of feature image.

- The scanning time for the proposed impact damage localization can be shortened by 10 times compared to that of the conventional method.

This work is the first attempt of applying PCA for impact damages on CFRP and we focus only on using a single $\mathrm{PC}$ to represent an area of impact damage. With a single PC as a feature, the data dimensions are extensively reduced to a 2-D information, therefore, the types of damages such as matrix cracks or delamination, which may occur within the layers are all flatten and characterized as an impact damage. Hence, the recommended future works include an investigation of using multiple PC features technique that facilitates damage characterization of different types, depth localization of delamination and matrix crack, investigation of Kernel PCA to improve nonlinear classification. 

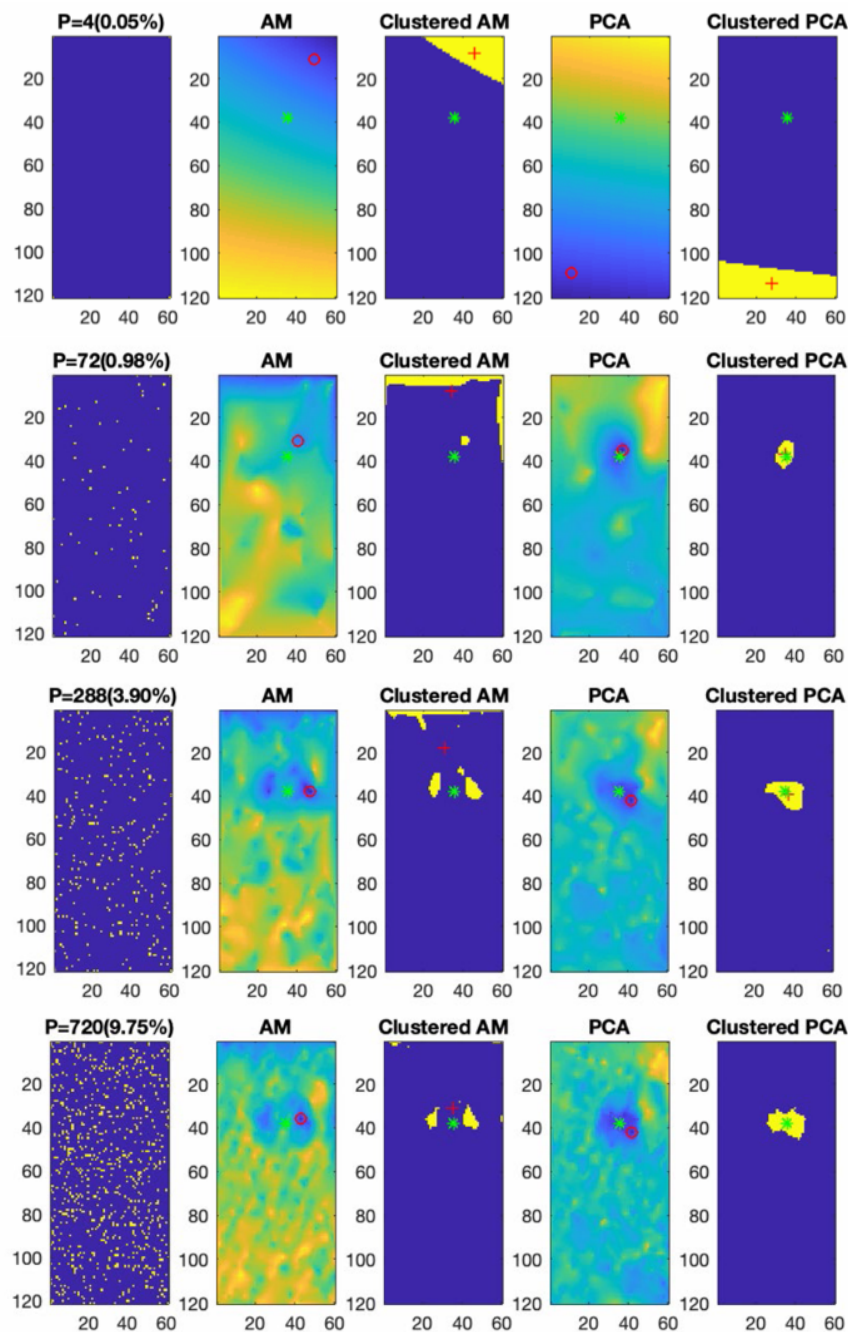

$*=$ actual impact location, $\mathrm{o}=$ minimum value, $+=$ centroid of cluster

Figure 4. Image reconstruction and locations of impact damage based on average magnitude (AM) and PCA feature with the number of sampling point $\mathrm{P}=4$ (first row), 72, 288 and 720 (last row).

Average distance error of AM and PCA features against number of points sampled

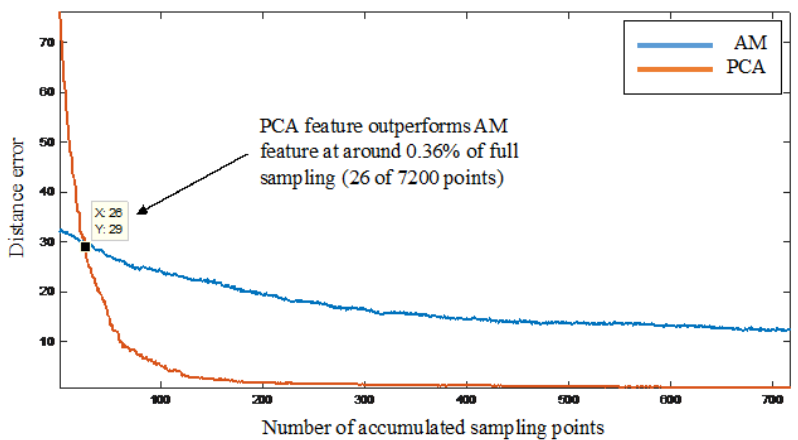

Figure 5. Average distance error of AM and PCA features 


\section{Acknowledgement}

The authors would like to thank the ministry of Science and Technology, Royal Thai government for the PhD scholarship support and acknowledge the "NDTonAIR" project for the research support.

\section{References}

[1] G. a. O. Davies, and R. Olsson, Impact on composite structures, The Aeronautical Journal. 108 (2004) 541-563. doi:10.1017/S0001924000000385.

[2] R. Zoughi, J.R. Gallion, and M.T. Ghasr, Accurate Microwave Measurement of Coating Thickness on Carbon Composite Substrates, IEEE Transactions on Instrumentation and Measurement. 65 (2016) 951953. doi:10.1109/TIM.2016.2526698.

[3] R. Sutthaweekul, G. Tian, Z. Wang, and F. Ciampa, Microwave open-ended waveguide for detection and characterisation of FBHs in coated GFRP pipes, Composite Structures. 225 (2019) 111080. doi:10.1016/j.compstruct.2019.111080.

[4] R. Sutthaweekul, G.Y. Tian, and M.D. Buhari, Detection of impact damage and fibre texture on CFRP using open-ended waveguide probe, in: 2016 13th International Conference on Electrical Engineering/Electronics, Computer, Telecommunications and Information Technology (ECTI-CON), 2016: pp. 1-6. doi:10.1109/ECTICon.2016.7561307.

[5] C. Tang, G.Y. Tian, K. Li, R. Sutthaweekul, and J. Wu, Smart Compressed Sensing for Online Evaluation of CFRP Structure Integrity, IEEE Transactions on Industrial Electronics. PP (2017) 1-1. doi:10.1109/TIE.2017.2698406.

[6] X. Yang, Y.R. Zheng, M.T. Ghasr, and K.M. Donnell, Microwave Imaging From Sparse Measurements for Near-Field Synthetic Aperture Radar, IEEE Transactions on Instrumentation and Measurement. PP (2017) 1-13. doi:10.1109/TIM.2017.2708379.

[7] R. Sutthaweekul, and G.Y. Tian, Steel Corrosion Stages Characterization Using Open-Ended Rectangular Waveguide Probe, IEEE Sensors Journal. 18 (2018) 1054-1062. doi:10.1109/JSEN.2017.2775521.

[8] A. Sophian, G.Y. Tian, D. Taylor, and J. Rudlin, A feature extraction technique based on principal component analysis for pulsed Eddy current NDT, NDT \& E International. 36 (2003) 37-41. doi:10.1016/S0963-8695(02)00069-5.

[9] L.I. Kuncheva, and W.J. Faithfull, PCA Feature Extraction for Change Detection in Multidimensional Unlabeled Data, IEEE Transactions on Neural Networks and Learning Systems. 25 (2014) 69-80. doi:10.1109/TNNLS.2013.2248094.

[10] H. Ledoux, and C. Gold, An Efficient Natural Neighbour Interpolation Algorithm for Geoscientific Modelling, in: P.F. Fisher (Ed.), Developments in Spatial Data Handling, Springer, Berlin, Heidelberg, 2005: pp. 97-108. doi:10.1007/3-540-26772-7_8.

[11] H. Ledoux, and C.M. Gold, Modelling three-dimensional geoscientific fields with the Voronoi diagram and its dual, International Journal of Geographical Information Science. 22 (2008) 547-574. doi:10.1080/13658810701517120.

[12] T. Celik, Unsupervised Change Detection in Satellite Images Using Principal Component Analysis and k-Means Clustering, IEEE Geoscience and Remote Sensing Letters. 6 (2009) 772-776. doi:10.1109/LGRS.2009.2025059.

[13] V. Lopresto, and G. Caprino, Damage Mechanisms and Energy Absorption in Composite Laminates Under Low Velocity Impact Loads, in: S. Abrate, B. Castanié, and Y.D.S. Rajapakse (Eds.), Dynamic Failure of Composite and Sandwich Structures, Springer Netherlands, 2013: pp. 209-289. doi:10.1007/978-94-007-5329-7_6. 